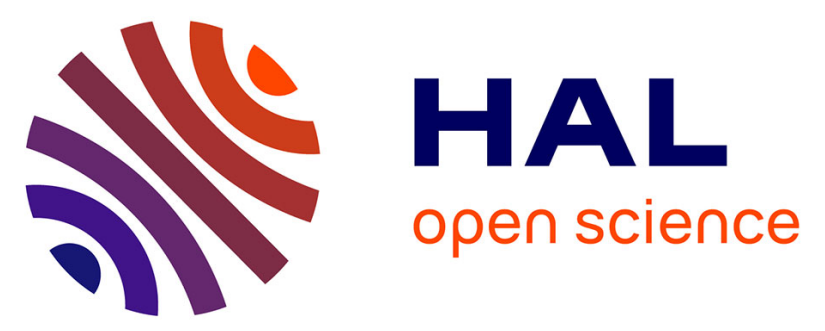

\title{
Hybrid Natural Element Method-Boundary Element Method for Unbounded Problems
}

Diego Pereira Botelho, Douglas Martins Araujo, Gérard Meunier, Yves Marechal, Jean-Louis Coulomb, Brahim Ramdane, Olivier Chadebec

\section{- To cite this version:}

Diego Pereira Botelho, Douglas Martins Araujo, Gérard Meunier, Yves Marechal, Jean-Louis Coulomb, et al.. Hybrid Natural Element Method-Boundary Element Method for Unbounded Problems. IEEE Transactions on Magnetics, 2015, 51 (3), 10.1109/TMAG.2014.2359140 . hal-02277681

\section{HAL Id: hal-02277681 \\ https://hal.science/hal-02277681}

Submitted on 26 Nov 2020

HAL is a multi-disciplinary open access archive for the deposit and dissemination of scientific research documents, whether they are published or not. The documents may come from teaching and research institutions in France or abroad, or from public or private research centers.
L'archive ouverte pluridisciplinaire HAL, est destinée au dépôt et à la diffusion de documents scientifiques de niveau recherche, publiés ou non, émanant des établissements d'enseignement et de recherche français ou étrangers, des laboratoires publics ou privés. 


\title{
Hybrid Natural Element Method - Boundary Element Method for Unbounded Problems
}

\author{
Diego Pereira Botelho ${ }^{1}$, Douglas Martins Araujo ${ }^{1,2}$, Gérard Meunier ${ }^{1}$, Yves Marechal ${ }^{1}$, Jean-Louis Coulomb ${ }^{1}$, Brahim \\ Ramdane $^{1}$ and Olivier Chadebec ${ }^{1}$ \\ ${ }^{1}$ Univ. Grenoble Alpes, G2Elab, F-38000 Grenoble, France \\ CNRS, G2Elab, F-38000 Grenoble, France \\ ${ }^{2}$ R\&D - Power Technology Strategy - Schneider Electric Industries SAS Grenoble, France
}

\begin{abstract}
This paper presents a hybrid approach coupling natural element method (NEM) and boundary element method (BEM) for the treatment of unbounded problems. The goal is to combine the accuracy of the NEM and the ability of the BEM in modeling linear and deformable domains without a mesh. The performance of the proposed scheme is compared to a FEM-BEM coupling in terms of accuracy. Results show that for a given number of degrees of freedom the proposed approach (NEM-BEM) is able to provide more accurate solutions.
\end{abstract}

Index Terms — boundary element method, coupled numerical methods, natural element method.

\section{INTRODUCTION}

$\mathrm{T}$ he Natural Element Method (NEM) is a numerical method based on the discretization by Voronoi diagram and on the concept of natural neighbors [1]. This method presents some characteristics of meshfree methods [2], like the highly accurate approximations and the shape functions with quasispherical influence zones. However, unlike most of the meshless methods, the NEM shape functions are interpolants, allowing the easy handling of the interface between materials and the direct imposition of essential boundary conditions. In terms of performance, the NEM has been proved to be more efficient (lower computational cost for a given accuracy) than the $1^{\text {st }}$ order finite element method (FEM) [3].

In this work, in order to model unbounded regions, we use an integral method known as the Boundary Element Method (BEM) [4]. Using this approach, the degrees of freedom are located on the interface between the domains (bounded and unbounded).

By combining the accuracy of the natural element method and the ability of the boundary element method in modeling linear and deformable domains without a mesh, the quality of interpolation on the magnetic material and of the field reconstitution in the unbounded region will be improved.

The developed approach is validated in the case of two 2D magnetostatic problems. The results are compared with analytical solution in the first case and with the classical finite element method for the second one.

\section{Magnetostatic PRoblem}

The 2D magnetostatic problem is defined by the Maxwell's equations:

$\nabla \times \boldsymbol{h}=\boldsymbol{j}$,

$\nabla \cdot \boldsymbol{b}=0$.

The representation of the modeled domains is showed in Fig. $1 . \Omega_{m}$ is the magnetic region (which can contain currents

Manuscript received June 6, 2014. Corresponding author: D. Martins Araujo (douglas.martins-araujo@fr.schneider-electric.com and magnets), $\Omega_{0}$ is the unbounded region (may optionally contain non-meshed coils) and $\Gamma_{m}$ is the interface between magnetic and unbounded domains.

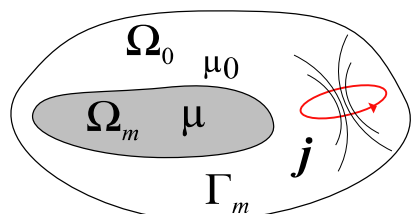

Fig. 1. Magnetostatic problem: bounded and unbounded regions.

In order to introduce the non-meshed coil a reduced magnetic scalar potential $\varphi_{m}$ is defined, as follow:

$\boldsymbol{h}=\boldsymbol{h}_{\text {red }}+\boldsymbol{h}_{0}$,
$\boldsymbol{h}_{\text {red }}=-\nabla \varphi_{m}$,

where $\boldsymbol{h}_{0}$ is the source field generated by the non-meshed coil and $\boldsymbol{h}_{\text {red }}$ is the reduced magnetic field.

\section{NATURAL ELEMENT METHOD}

The natural element method uses the concept of natural neighbors. It is based on the construction of Voronoï diagram on a cloud of nodes. For a set of nodes $N=\left\{n_{1}, n_{2}, n_{3} \ldots n_{N}\right\}$ distributed in a given 2-D domain, the Voronoï diagram is a subdivision of the domain into cells, where each cell $C_{i}$ associated to node $n_{i}$ is such that any point in $C_{i}$ is closer to node $n_{i}$ than to any other node $n_{j}$ for $i \neq j$. In mathematical terms, a Voronoï cell is defined as:

$C_{i}=\left\{\boldsymbol{x} \in \mathfrak{R}^{n}, d\left(\boldsymbol{x}, \boldsymbol{x}_{\boldsymbol{i}}\right)<d\left(\boldsymbol{x}, \boldsymbol{x}_{\boldsymbol{j}}\right), \forall j \neq i\right\}$,

where $d$ is the distance between two points in Euclidean metric.

Fig. 2 illustrates a Voronoï cell related to a node $n_{i}$ in a 2-D space. The Delaunay triangulation, which is the dual of the Voronoï diagram, is constructed by connecting the nodes whose Voronoï cells have common boundaries. These nodes are called natural neighbors. Alternatively, the vertices of the 
Voronoï cell are the center of the circumcircles of the Delaunay triangles.

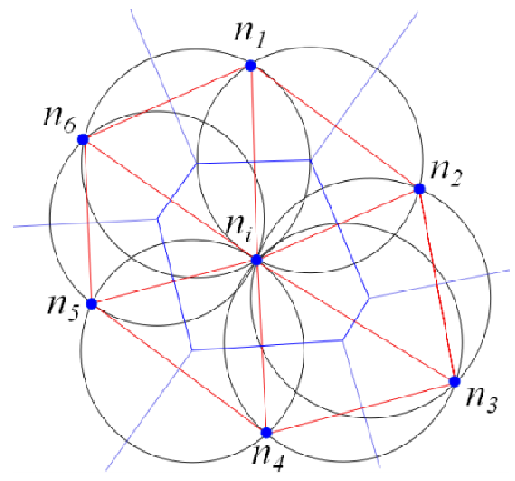

Fig. 2. Voronoï diagram in blue, its associated Delaunay triangulation in red and the circumcircles associated to the Delaunay triangles in grey. Around $n_{i}$, its Voronoï cell and its 6 natural neighbors, from $n_{1}$ to $n_{6}$.

\section{A. Sibson interpolation}

Data interpolation based on Voronoï diagrams can be obtained through different techniques [5]. In this work, we will describe the scheme proposed by Sibson [6] in 1980. For sake of simplicity, we will consider a cloud of nodes with known nodal values in a 2-D domain, and look for the interpolated value at an evaluation point $\boldsymbol{x}$.

Initially, the evaluation point $\boldsymbol{x}$ is added to the "original" Voronoï diagram and a new Voronoï cell is created around it (Fig. 3). The natural neighbors of $\boldsymbol{x}$ (i.e. the closest nodes) are the nodes that share a Voronoï edge with the Voronoï cell of $\boldsymbol{x}$. These will be the nodes used for the interpolation at the evaluation point.

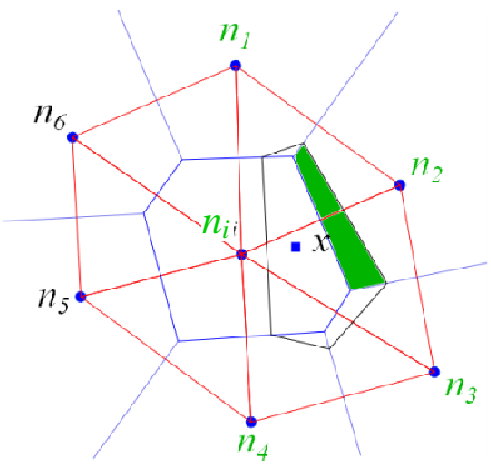

Fig. 3. Sibson natural neighbors and natural coordinates (original Vorono diagram in blue, natural neighbors in green, additional Voronoï cell due to $\boldsymbol{x}$ in black and intersection area between Voronoï cells of $\boldsymbol{x}$ and $n_{2}$ in green).

The influence of each natural neighbor on the evaluation point through Sibson's technique is given by (6), where each $A_{i}(\boldsymbol{x})$ represents the intersection area between the Voronoï cell centered on $x$ and the "original" Voronoï cell related to the natural neighbor $n_{i}$, as illustrated by the hashed region in Fig. 3.

$$
\alpha_{i}(x)=\frac{A_{i}(x)}{\sum_{j} A_{j}(x)}
$$

It is easy to verify that the Sibson shape functions (6) are always positive and less than 1 and that the sum of all terms is equal to 1 . One can equally remark that when $\boldsymbol{x}$ tends towards $n_{i}$, the shape function tends towards 1 for node $n_{i}$ and towards 0 for all other nodes (therefore the function is interpolant).

The interpolating character of the Sibson shape functions allows the easy application of the boundary conditions. Furthermore, the shape functions degenerate to a simple linear interpolation at the borders [1]. Fig. 4 (a) depicts the Sibson shape function related to a node. Its support is given by the union of all circumcircles passing through this node.

Last, Fig. 4 Erreur ! Source du renvoi introuvable.(b) shows the elevation of the Sibson shape function on a regular grid of nodes.
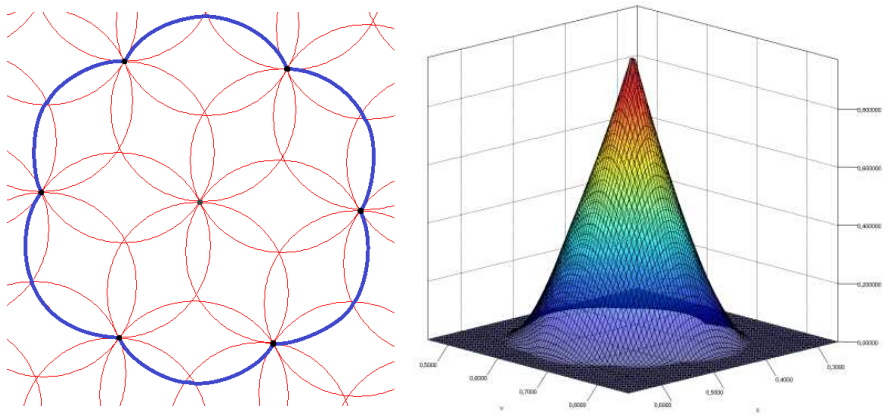

Fig. 4. (a) Support for NEM shape function and (b) Plot of the Sibson shape function.

\section{B. Galerkin formulation}

In order to solve (2), the reduced scalar magnetic potential is used by combine (2), (3) and (4). This potential is interpolated using (6). Then, by applying the Galerkin method to the resulted equation and considering a constant permeability in $\Omega_{m}$, we get:

$\int_{\Omega_{m}} \nabla \alpha_{i} \mu \nabla \alpha_{j} \varphi_{m j} d \Omega+\int_{\Gamma_{m}} \alpha_{i} \alpha_{j} b_{n j} d \Gamma=\int_{\Gamma_{m}} \alpha_{i} \mu H_{0 n} d \Gamma$,

where $\varphi_{m}$ is the reduced magnetic potential, $b_{n}$ is the magnetic induction normal to the boundary and $H_{0 n}$ is the source magnetic field normal to the boundary.

In order to simplify the notation, (7) can be rewritten as

$[S] \cdot \varphi_{m}+[\boldsymbol{U}] \cdot \boldsymbol{b}_{n}=\boldsymbol{Q}$,

with

$S_{i j}=\int_{\Omega_{m}} \nabla \alpha_{i} \mu \nabla \alpha_{j} d \Omega, \quad U_{i j}=\int_{\Gamma_{m}} \alpha_{i} \alpha_{j} d \Gamma$ and $Q_{i j}=\int_{\Gamma_{m}} \alpha_{i} \mu H_{0 n} d \Gamma$

\section{BOUNDARY ELEMENT METHOD (BEM)}

If no source field in the free space is taken into account, the problem can be represented by a Laplacian equation in term of a total scalar magnetic potential. The main idea is to solve Laplacian equation transforming the volume integral 
equation over $\Omega_{0}$ into a surface integral at the boundary $\Gamma_{m}$. The classical boundary integral equation is obtained by using the third Green's identity [4].

Since we have non-meshed coil in the air, the reduced magnetic potential $\varphi_{\Gamma}$ will be introduced. According to (3), we get:

$$
\begin{aligned}
& c\left(\boldsymbol{x}_{\boldsymbol{0}}\right) \varphi_{\Gamma}\left(\boldsymbol{x}_{\boldsymbol{\theta}}\right)= \\
& \quad \int_{\Gamma_{m}}\left(\nabla_{n} G\left(\boldsymbol{x}_{\boldsymbol{0}}, \boldsymbol{x}\right) \varphi_{\Gamma}-G\left(\boldsymbol{x}_{\boldsymbol{0}}, \boldsymbol{x}\right)\left(\frac{1}{\mu_{0}} \boldsymbol{b}-\boldsymbol{h}_{\boldsymbol{0}}\right) \cdot \boldsymbol{n}\right) d \Gamma,
\end{aligned}
$$

where $G\left(\boldsymbol{x}_{0}, \boldsymbol{x}\right)$ is the Green's function $\ln (1 / r), r$ is the distance between $\boldsymbol{x}_{0}$ and a point $\boldsymbol{x}$ on the boundary and $c\left(\boldsymbol{x}_{0}\right)$ is the solid angle subtended by the boundary $\Gamma_{m}$ at $\boldsymbol{x}_{0}$.

The interpolation of the potential and the magnetic induction normal to the boundary are given by:

$\boldsymbol{\varphi}_{\Gamma}(\boldsymbol{x})=\sum_{j}^{N_{\Gamma}} w_{j}(\boldsymbol{x}) \varphi_{\Gamma j}, b_{n}(\boldsymbol{x})=\sum_{j}^{N_{\Gamma}} w_{j}(\boldsymbol{x}) b_{n_{j}}$,

where $N_{\Gamma}$ the number of facets of the boundary and $w_{j}(\boldsymbol{x})=1$ if $\boldsymbol{x}$ belongs to the boundary element $j$ and $w_{j}(\boldsymbol{x})=0$ otherwise (0-order). Equation (11) can be rewritten, as follow:

$[\boldsymbol{H}] \varphi_{\Gamma}+[\boldsymbol{T}] \boldsymbol{b}_{\boldsymbol{n}}=\left[\boldsymbol{T}_{1}\right] \boldsymbol{h}_{0 \boldsymbol{n}}$

$\boldsymbol{T}, \boldsymbol{T}_{\mathbf{1}}$ and $\boldsymbol{H}$ matrices are defined as follows:

$$
\begin{aligned}
& T_{i j}=\frac{1}{\mu_{0}} \int_{\Gamma_{m_{j}}} G\left(\boldsymbol{x}_{\boldsymbol{i}}, \boldsymbol{x}_{\boldsymbol{j}}\right) d \Gamma, T_{1 i j}=\int_{\Gamma_{m_{j}}} G\left(\boldsymbol{x}_{\boldsymbol{i}}, \boldsymbol{x}_{\boldsymbol{j}}\right) d \Gamma, \\
& H_{i j}=c_{i i}-\int_{\Gamma_{m_{j}}} \nabla_{n} G\left(\boldsymbol{x}_{\boldsymbol{i}}, \boldsymbol{x}_{\boldsymbol{j}}\right) d \Gamma,
\end{aligned}
$$

where $c_{i i}$ is equals $\pi$, since the collocation point is located on the centroid of each boundary element.

There are many ways to perform integration of (13). In this work the well-known analytical expression found in [7] has been used.

\section{COUPLING Both MethodS}

By considering conservation of the normal component of the magnetic induction and the unicity of the reduced magnetic potential on boundary, we get [8]:

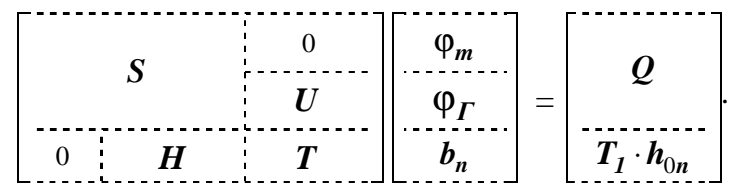

It's important to remark that the BEM portion of the matrix is fully populated and the NEM portion is banded. Moreover the equation (14) leads to a linear system which will be solved directly.

By solving the system above, we get the reduced scalar magnetic potential on all nodes and $\boldsymbol{b}_{\boldsymbol{n}}$ on the boundary [9].

\section{RESULTS}

The validation of the presented scheme was performed through the simulation of two test cases.

\section{A. Results on a test case with analytical solution}

Fig. 5 shows a homogeneous infinite cylinder under the influence of a constant magnetic field $\left(\boldsymbol{H}_{0}=1 \mathrm{~A} / \mathrm{m}\right)$. The magnetic domain (cylinder) is modeled using NEM and the unbounded domain (air) using BEM.

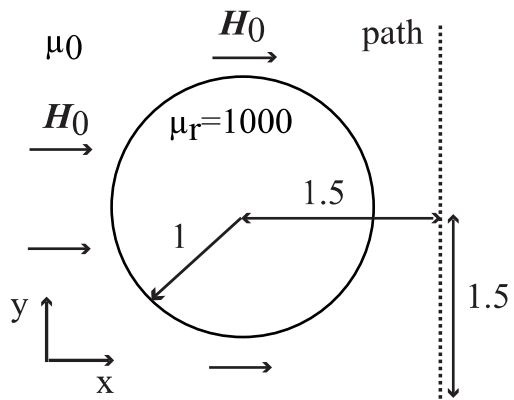

Fig. 5. Infinite cylinder with a constant permeability immersed in a constant field $\boldsymbol{H}_{0}$. The total field is evaluated on the path.

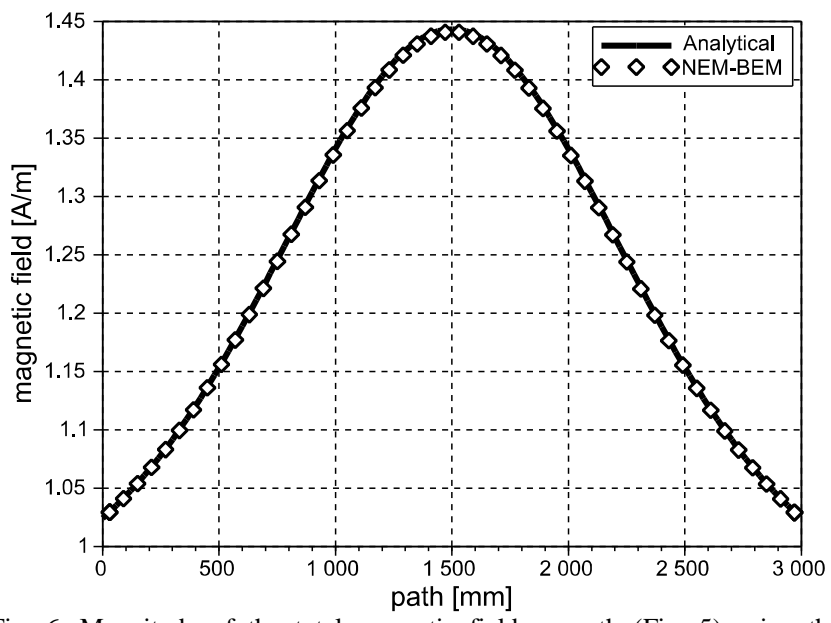

Fig. 6. Magnitude of the total magnetic field on path (Fig. 5) using the cylinder problem for both analytical and NEM-BEM approach.

The problem was solved with a relatively coarse discretization, generating 232 degrees of freedom (which 80 were on the boundary). Once the problem is solved, the integral equation (11) is used to evaluate the reduced scalar magnetic potential at the path showed in Fig. 5. Then, since the source field is known, by (3) and (4) the total magnetic field is evaluated. More details on the evaluating of the total magnetic field can be found in [10].

Fig. 6 shows the comparison between the analytical result and the NEM-BEM one. A good agreement between the solutions is verified.

\section{B. Results on a real case}

Fig.7 shows a magnetic circuit with a non-meshed coil. In order to test the robustness of the proposed scheme the magnetic induction was computed on two paths: the first one in the gap between the parts of the device and the second one crossing the magnetic circuit.

Once there is no exact analytical solution to this problem, a $2^{\text {nd }}$ order FEM solution was used as reference. This solution 
was computed with a very fine mesh.

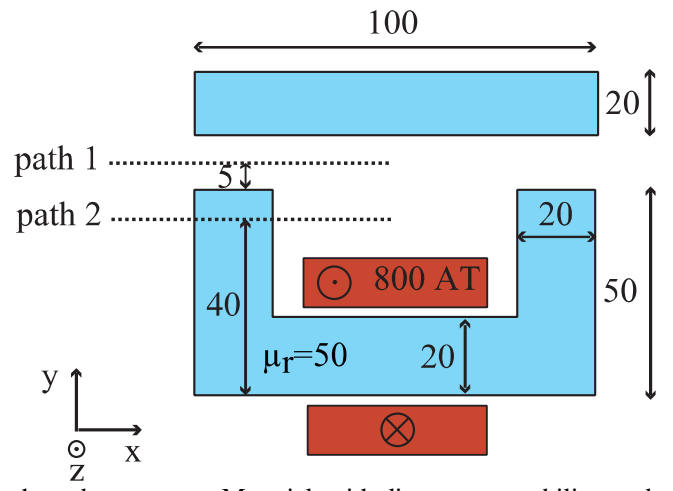

Fig. 7. U shaped contactor. Material with linear permeability and a nonmeshed coil. Two different paths have been considered.

Three different levels of discretization where tested in order to test the convergence of the proposed method. Table 1 shows the number of degrees of freedom (DoF) for each case.

Fig. 8 presents the comparison between the tested cases and the reference.

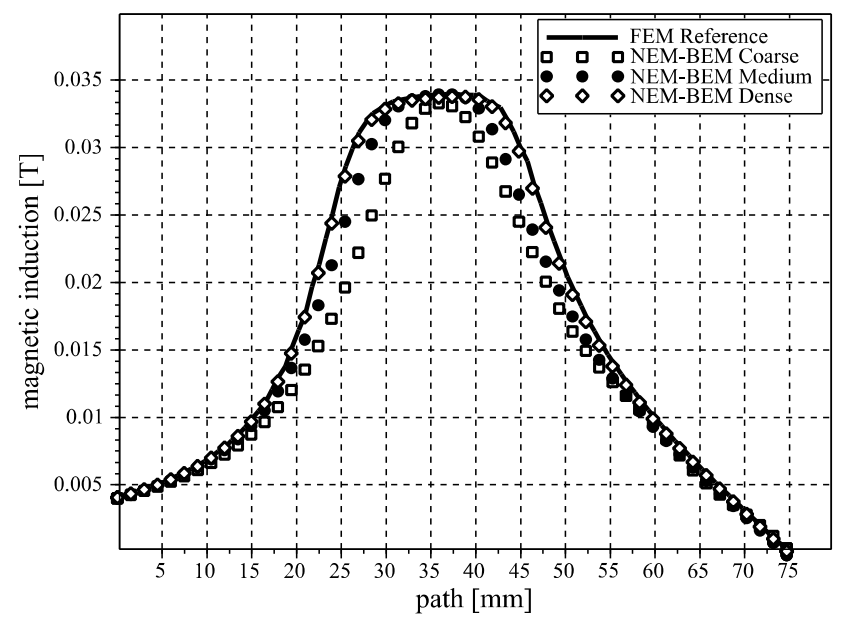

Fig. 8. Comparison between FEM and the approach presented. Vertical component of the magnetic field on path 1 .

Results show a fast convergence to the reference value as the number of DoF increases. One can remark that the number of DoF required to get an accurate result is much lower than the one of the reference as shown in table I.

TABLE 1

DEGREES OF FREEDOM

\begin{tabular}{|c|c|c|c|c|}
\hline & $\begin{array}{c}\text { FEM 2nd } \\
\text { Reference }\end{array}$ & $\begin{array}{c}\text { NEM-BEM } \\
\text { Dense }\end{array}$ & $\begin{array}{c}\text { NEM-BEM } \\
\text { Medium }\end{array}$ & $\begin{array}{c}\text { NEM-BEM } \\
\text { Coarse }\end{array}$ \\
\hline DoF & 129,751 & 3,745 & 265 & 77 \\
\hline
\end{tabular}

Fig. 9 illustrates the magnetic induction on the path 2 obtained by NEM-BEM and FEM-BEM. Let us notice that both solutions use the same number of DoF $(4,512)$ and exactly the same node distribution.

A good agreement between the two methods and the reference is achieved. However, one can observe that the NEM-BEM provides a much smoother approximation when compared to FEM-BEM.

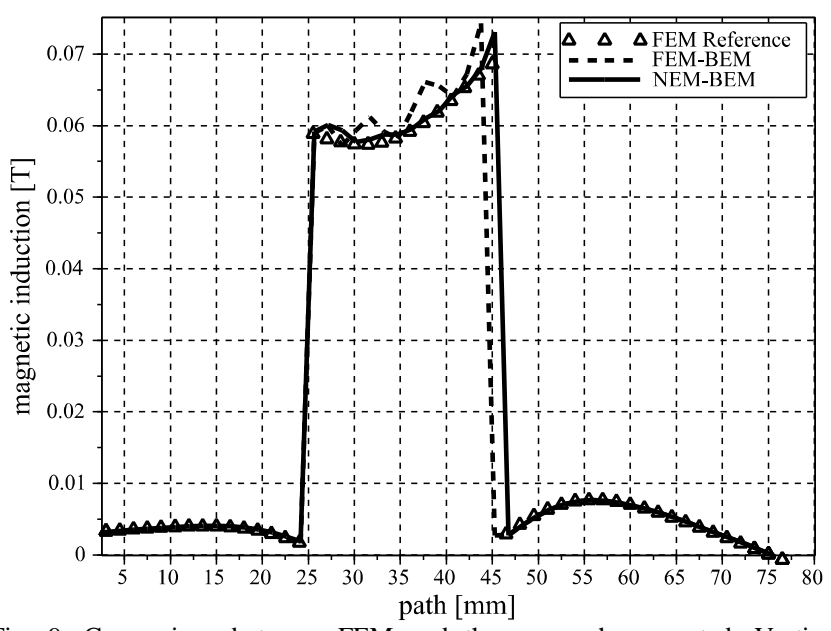

Fig. 9. Comparison between FEM and the approach presented. Vertical component of the magnetic field on path 2 .

\section{CONCLUSION}

In this paper, an original hybrid natural element method boundary element method formulation has been presented. This scheme is very useful, once the flux linkage is automatically taken into account and the mesh dependency is drastically reduced.

The NEM-BEM was able to provide accurate solutions even with very coarse discretizations. Besides that, this new formulation is attractive because of the easiness on the treatment of the unbounded regions and the smoothness of the interpolation inside magnetic regions.

\section{REFERENCES}

[1] N. Sukumar, "The natural element method in solid mechanics," Northwestern University, 1998.

[2] T. Belytschko, T. Rabczuk, A. Huerta, and S. Fernández-Méndez, "Meshfree Methods," in Encyclopedia of Computational Mechanics, John Wiley \& Sons, Ltd, 2004.

[3] Y. Maréchal, B. Ramdane, and D. Pereira Botelho, "Computational Performances of Natural Element and Finite Element Methods," Magnetics, IEEE Transactions on, vol. 50, no. 2, pp. 405-408, 2014.

[4] C. A. Brebbia, The Boundary Element Method For Engineers. Pentech Press, 1984.

[5] E. Cueto, N. Sukumar, B. Calvo, M. A. Martínez, J. Cegonino, and M. Doblaré, "Overview and recent advances in natural neighbour Galerkin methods," Archives of computational methods in engineering, vol. 10, no. 4, pp. 307-384, 2003.

[6] R. Sibson, "A vector identity for the Dirichlet tessellation," in Mathematical Proceedings of the Cambridge Philosophical Society, 1980, vol. 87, pp. 151-155.

[7] J. Wang and T.-K. Tsay, "Analytical evaluation and application of the singularities in boundary element method," Engineering Analysis with Boundary Elements, vol. 29, no. 3, pp. 241-256, Mar. 2005.

[8] T. Eibert and V. Hansen, "Calculation of unbounded field problems in free space by a 3D FEM/BEM-hybrid approach," Journal of Electromagnetic Waves and Applications, vol. 10, no. 1, pp. 61-78, 1996.

[9] G. Meunier, J.-L. Coulomb, S. J. Salon, and L. Krahenbul, "Hybrid finite element boundary element solutions for three dimensional scalar potential problems," IEEE Transactions on Magnetics, vol. 22, no. 5, pp. 1040-1042, 1986

[10] H. Ma and N. Kamiya, "A general algorithm for accurate computation of field variables and its derivatives near the boundary in BEM," Engineering Analysis with Boundary Elements, vol. 25, no. 10, pp. 833-841, décembre 2001. 